\title{
Cardiac troponin I measured with a very high sensitivity assay predicts subclinical carotid atherosclerosis: The Akershus Cardiac Examination 1950 Study
}

\author{
Magnus Nakrem Lyngbakken ${ }^{\mathrm{a}, \mathrm{b}}$, Thea Vigen ${ }^{\mathrm{b}, \mathrm{c}}$, Håkon Ihle-Hansen ${ }^{\mathrm{b}, \mathrm{d}}$, Jon Brynildsen ${ }^{\mathrm{a}, \mathrm{b}}$, \\ Trygve Berge $^{\mathrm{b}, \mathrm{d}}$, Ole Morten Rønning ${ }^{\mathrm{b}, \mathrm{c}}$, Arnljot Tveit ${ }^{\mathrm{b}, \mathrm{d}}$, Helge Røsjø ${ }^{\text {b,e }}$, Torbjørn Omland ${ }^{\mathrm{a}, \mathrm{b}, "}$

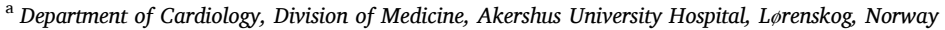 \\ ${ }^{\mathrm{b}}$ Institute of Clinical Medicine, University of Oslo, Oslo, Norway \\ ${ }^{\mathrm{c}}$ Department of Neurology, Division of Medicine, Akershus University Hospital, Lorenskog, Norway \\ d Department of Medical Research, Barum Hospital, Vestre Viken Hospital Trust, Norway \\ ${ }^{\mathrm{e}}$ Division of Research and Innovation, Akershus University Hospital, Lorenskog, Norway
}

\section{A R T I C L E I N F O}

\section{Keywords:}

Cardiac troponin

Carotid atherosclerosis

Risk prediction

Population studies

\begin{abstract}
A B S T R A C T
Aims: Concentrations of cardiac troponin I (cTnI) are associated with incident ischemic stroke and predict the presence and severity of coronary atherosclerosis. Accordingly, we hypothesized that concentrations of cTnI measured with a very high sensitivity (hs-) assay would be associated with subclinical stages of carotid atherosclerosis in the general population.

Methods: We measured hs-cTnI on the Singulex Clarity System in 1745 women and 1666 men participating in the prospective observational Akershus Cardiac Examination 1950 Study. All study participants were free from known coronary heart disease and underwent extensive cardiovascular phenotyping at baseline, including carotid ultrasound. We quantified carotid atherosclerosis by the carotid plaque score, carotid intima-media thickness (cIMT) and the presence of hypoechoic plaques.

Results: Concentrations of hs-cTnI were measurable in $99.8 \%$ of study participants and were significantly associated with increased carotid plaque score (odds ratio for quartile 4 of hs-cTnI 1.59, 95\% CI 1.22 to 2.07 , p for trend $<0.001$ ) and cIMT (odds ratio for quartile 4 of hs-cTnI 1.57, 95\% CI 1.02 to 2.42, p for trend $=0.036$ ), but not with the presence of hypoechoic plaques. hs-cTnI concentrations significantly improved reclassification and discrimination models in predicting carotid plaques when added to cardiovascular risk factors, no improvements were evident in predicting cIMT or hypoechoic plaques.

Conclusion: Concentrations of cTnI measured with a very high sensitivity assay are predictive of carotid atherosclerotic burden, a phenomenon likely attributable to common risk factors of subclinical myocardial injury, coronary and carotid atherosclerosis.
\end{abstract}

\section{Introduction}

Measurement of cardiac troponin is essential in the diagnostic workup of patients with suspected acute coronary syndrome and the advent of high sensitivity (hs-) cardiac troponin assays permits safe and swift diagnosis of acute myocardial infarction. More recently, evidence is amassing for the use of cardiac troponin measured with hs-assays in predicting incident cardiovascular disease such as myocardial infarction [1], heart failure [2], atrial fibrillation [3], and ischemic stroke [4]. Concentrations of cardiac troponin also increase after ischemic stroke, causes of which are largely multifactorial [5].

Atherosclerosis is a chronic inflammatory disease affecting large and medium sized arteries of the entire body, principally resulting in ischemia of the brain, heart and extremities. Non-invasive imaging procedures like carotid ultrasonography and computer tomography may identify subclinical atherosclerosis and convey prognostic information beyond established risk prediction models [6]. Of the two, carotid ultrasonography might be considered the preferred initial screening strategy, being both safe and radiation free.

In subjects recruited from the general population, carotid

\footnotetext{
* Corresponding author at: Department of Cardiology, Akershus University Hospital, Postboks 1000, 1478 Lørenskog, Oslo, Norway.

E-mail address: torbjorn.omland@medisin.uio.no (T. Omland).
} 
atherosclerotic burden is associated with increased concentrations of cardiac troponin I (cTnI) measured with contemporary assays [7]. Concentrations of hs-cTnI also appear to be associated with reduced large vessel vascular function [8]. Additionally, detectable concentrations of hs-cardiac troponin $\mathrm{T}$ (hs-cTnT) are associated with the presence of carotid plaques in patients with systemic lupus erythematous [9] and with vulnerable coronary lesions in patients with stable angina [10].

Established cardiovascular risk factors such as diabetes mellitus and hypertension are associated with all forms of atherosclerosis, as well as increased concentrations of cardiac troponin [1], possibly providing a link between subclinical atherosclerosis and low-grade cardiac troponin release. No studies have assessed the associations between cTnI measured with a very high sensitivity assay and subclinical carotid atherosclerosis in the general population. Given correlations between cardiac troponin concentrations and indices of carotid atherosclerosis, this information could prove valuable to guide early preventive interventions in subjects with subclinical disease.

Accordingly, in subjects without previous manifestation of atherosclerotic cardiovascular disease, we hypothesized that (1) concentrations of hs-cTnI are associated with subclinical carotid atherosclerosis, and that (2) concentrations of hs-cTnI provide incremental information to established cardiovascular risk factors in assessing subclinical carotid atherosclerosis.

\section{Methods}

\subsection{Study overview}

The Akershus Cardiac Examination (ACE) 1950 Study is a prospective, population-based cohort of 3706 individuals from Akershus County, Norway. All community dwellers born in 1950 residing in Akershus County were invited to participate $(n=5827)$, and baseline examinations were conducted September 2012 to May 2015. The study was conducted at the two hospitals of Akershus County, Norway: Akershus University Hospital and Bærum Hospital/Vestre Viken Hospital Trust. The study design has previously been reported [11]. The study complies with the Declaration of Helsinki and has been approved by the Regional Committee for Medical Research Ethics. All participants provided informed written consent before study commencement.

\subsection{Participants}

Data on medical history, current medication and socioeconomic status, as well as data on alcohol and tobacco consumption, are selfreported. Higher education was defined as education at the level of college, university or equivalent. Co-morbidities were defined as follows: coronary artery disease (CAD; self-reported history of myocardial infarction, coronary artery bypass grafting or percutaneous coronary intervention), and diabetes mellitus (self-reported diabetes, daily use of antidiabetic medication, or biochemical evidence supporting the diagnosis, i.e. elevated concentrations of $\mathrm{HbA1c}[\geq 6.5 \%]$ and fasting blood glucose $[\geq 7.0 \mathrm{mmol} / \mathrm{L}]$ on baseline examination). Participants with known CAD at baseline $(\mathrm{n}=263)$ were excluded from the analyses, as well as participants with missing carotid ultrasound examination ( $\mathrm{n}=$ 21 ) or biomarker data $(n=11)$.

\subsection{Carotid ultrasonography}

A detailed description of the carotid ultrasonography examination has been published previously [12]. Briefly, ultrasound images of the right and left extracranial arteries (common carotid artery, internal and external carotid artery) were recorded in both long- and short-axis views on the GE Vivid E9. We assessed three different carotid ultrasound indices of subclinical atherosclerosis; quantitative carotid plaque burden (carotid plaque score), carotid intima media thickness (cIMT), and prevalent hypoechoic plaques [13]. For the carotid plaque score, the carotid artery was divided into four segments (common carotid artery, bifurcation, and internal and external carotid artery), and plaques, as defined by Mannheim Carotid Intima Media Thickness and Plaque Consensus, were assessed in each segment on both sides. Each segment was scored according to the diameter of the thickest plaque present ( $\geq 1.5 \mathrm{~mm}=1$ point, $\geq 2.5 \mathrm{~mm}=2$ points, and $\geq 3.5 \mathrm{~mm}=3$ points), totaling a maximum plaque score of 24 points. cIMT was measured in Bmode and in a lateral view using a semiautomatic tracking system in the far wall of the common carotid artery and in a region free from plaque. We used the mean average cIMT value from the left and right common carotid artery. Plaque morphology was registered as presence of one or more hypoechoic plaques. To identify hypoechoic plaques, the vessel lumen was used as reference structure [14]. The ultrasound examinations were performed by two ultrasound technicians and two physicians, all trained in vascular ultrasound, and all underwent a joint training program before the start of the study. The analyses were performed offline on Echopac PC v12 (GE Vingmed) by the two physicians. Interand intrarater reliability tests were performed twice during the inclusion period, in which the two physicians measured plaque diameter twice for plaque score calculation on the same 25 randomly selected examinations. Both tests showed excellent results (Cronbach $\kappa=0.999$ ).

\subsection{Blood sampling procedures and biochemical assays}

Fasting venous blood samples were centrifuged at room temperature and frozen at $-80{ }^{\circ} \mathrm{C}$. Analyses of C-reactive protein (CRP, measured with a high sensitivity assay), glucose, glycated hemoglobin (HbA1c), cholesterol (total, high-density lipoprotein [HDL]), triglycerides, and creatinine, were performed immediately by routine hospital laboratory clinical chemistry. We estimated concentrations of LDL cholesterol using the Friedewald equation [15]. CRP concentrations above or equal to 3 $\mathrm{mg} / \mathrm{L}$ were used to denote the presence of low-grade systemic inflammation. Estimated glomerular filtration rate (eGFR) was estimated with the Chronic Kidney Disease Epidemiology Collaboration equation. cTnI was measured in 2017 from thawed serum samples using a novel very hs-assay (hs-cTnI) on the Singulex Clarity System. The coefficient of variation (CV) for this assay has been reported to be $10 \%$ at $0.53 \mathrm{ng} / \mathrm{L}$ and $20 \%$ at $0.14 \mathrm{ng} / \mathrm{L}$ [16]. Using control material from Singulex, we found a CV of $11.3 \%$ at 3.85 (standard deviation [SD] 0.44) ng/L, $10.5 \%$ at 10.37 (SD 1.09) $\mathrm{ng} / \mathrm{L}, 7.3 \%$ at 26.37 (SD 1.92) ng/L, and $7.8 \%$ at 8239.62 (SD 645.92) ng/L. The limit of detection for this assay is 0.08 $\mathrm{ng} / \mathrm{L}$, with a 99th percentile value in a reference population of $6.74 \mathrm{ng} / \mathrm{L}$ [16]. Concentrations below the limit of detection were assigned a value of $50 \%$ of the limit of detection (i.e. $0.04 \mathrm{ng} / \mathrm{L}$ ).

\subsection{Statistical methods}

Baseline data are reported as absolute numbers (proportion) or median (interquartile range) unless otherwise stated. Continuous variables were analyzed with the Mann-Whitney $U$ test, and categorical variables were analyzed with the Fisher exact test. Correlations were assessed by Spearman's rank correlation coefficient. The associations between carotid ultrasound variables and hs-cTnI were assessed by logistic regression models constructed on guideline recommended cut-offs for cIMT $(>0.90 \mathrm{~mm}$ ) [17], and the presence of hypoechoic plaques [18]. There is currently no guideline based consensus for calculation of carotid plaque score [19]. For the plaque score utilized in the current investigation, a value $\geq 4$ is associated with increased cardiovascular risk and higher prevalence of cardiovascular risk factors [12], and this cut-off was accordingly used for the logistic regression models in predicting carotid plaques.

All regression models were incrementally adjusted for sex, age, study site, and a priori selected variables influencing cardiovascular risk (BMI, eGFR, total, HDL and LDL cholesterol, CRP, higher education, systolic blood pressure, diabetes mellitus, current smoking, and alcohol consumption). Adjustment was also made for current statin therapy, as 
statin therapy is associated with attenuation of both cardiac troponin concentrations [20] and atherosclerosis [21]. Participants with missing covariate data were excluded from the multivariable regression analyses. The incremental value of hs-cTnI to established cardiovascular risk factors was assessed by $\mathrm{C}$ statistics derived from the logistic regression models, as well as by the net reclassification index (NRI) and integrated discrimination improvement (IDI). We compared overall model fit by the likelihood-ratio test. We additionally performed analysis stratified according to sex, as both cardiovascular risk [22,23] and concentrations of cardiac troponin [24] exhibit sex specific discrepancies. Statistical significance was assumed at $\mathrm{p}<0.05$. The analyses were performed with STATA 16 (StataCorp LP, College Station, TX).

\section{Results}

\subsection{Baseline characteristics}

Of the 3706 participants from the ACE1950 Study baseline examination, 1745 women and 1666 men were included in the following analyses (Fig. 1). Characteristics of participants excluded because of a history of CAD $(n=263)$ or missing data for carotid ultrasonography ( $\mathrm{n}$ $=21)$ or hs-cTnI $(n=11)$ are summarized in Supplementary Table 1 . Excluded participants were more frequently male with diabetes mellitus and higher BMI. They were more frequently on cardioprotective and antidiabetic medication, and exhibited increased concentrations of hscTnI, as well as a more unfavorable metabolic profile.

Concentrations of hs-cTnI were measurable in $99.8 \%$ of study participants (99.9\% in women, $99.8 \%$ in men; $p=0.44$; Fig. 2). Patient characteristics according to quartiles of hs-cTnI are summarized in Table 1. Compared to participants in the lowest quartile, participants in the upper quartile of hs-cTnI were more frequently male with diabetes mellitus, had higher blood pressure, BMI, carotid plaque score and cIMT, lower eGFR, and were more frequently on cardioprotective medication. They were also less frequently current smokers.

\section{2. hs-cTnI and carotid indices of subclinical atherosclerosis}

Continuous concentrations of hs-cTnI correlated significantly with carotid plaque score $(\mathrm{r}=0.16 ; \mathrm{p}<0.001)$ and cIMT $(\mathrm{r}=0.14 ; \mathrm{p}<$ $0.001)$, but not hypoechoic plaques $(r=0.03 ; p=0.08)$. In adjusted analyses according to quartiles of hs-cTnI, increasing concentrations of hs-cTnI were associated with increased carotid plaque score ( $\mathrm{p}$ for trend $<0.001$ ) and cIMT (p for trend $=0.036$ ), but not with the presence of hypoechoic plaques ( $\mathrm{p}$ for trend $>0.99$; Table 2).

\subsection{Discrimination and reclassification}

Table 3 shows discrimination and reclassification models of hs-cTnI for identifying study participants with increased carotid plaque score and cIMT, and the presence of hypoechoic plaques. The basic risk model comprised established cardiovascular risk factors and demographics, and the distributions of the predicted risk probabilities of the basic models are demonstrated in Supplementary Fig. 1. Participants in the 4th quartile of predicted risk probabilities had higher concentrations of hs-cTnI for all three risk prediction models (Supplementary Table 2). Compared to the basic risk model, the addition of hs-cTnI improved overall model fit ( $\mathrm{p}$ for likelihood-ratio test $<0.001$ ) and C statistics ( $0.706,95 \%$ confidence interval [CI] 0.685 to 0.726 vs $0.710,95 \%$ CI 0.690 to $0.730 ; \mathrm{p}=0.049$ ) in predicting carotid plaques. NRI was improved (0.171, $95 \%$ CI 0.084 to 0.247 ) with higher diagnostic probabilities to participants with carotid plaque score $\geq 4$, and lower diagnostic probabilities to participants with carotid plaque score $<4$. Similarly, IDI was improved (0.003, 95\% CI 0.000 to 0.009 ) with improvement in discrimination slope and corresponding improvements in model sensitivity and specificity. The addition of hs-cTnI to the basic risk model improved overall model fit ( $\mathrm{p}$ for likelihood-ratio test 0.035 ) for cIMT, but apart from this, no improvements were evident in predicting cIMT or hypoechoic plaques.

\subsection{Impact of sex}

The associations between hs-cTnI and carotid indices of subclinical atherosclerosis according to sex are presented in Supplementary Table 3. The associations between hs-cTnI and carotid plaque burden were numerically stronger for women (odds ratio for women 1.90, 95\% CI 1.25 to 2.88 vs. odds ratio for men 1.29 , $95 \%$ CI 0.88 to 1.88 ), and numerically stronger for cIMT for men (odds ratio for women 1.05, $95 \%$ CI 0.49 to 2.28 vs. odds ratio for men 2.20 , $95 \%$ CI 1.19 to 4.10 ). hs-cTnI was not associated with hypoechoic plaques for either sex. There was no effect modification by sex on the associations between hs-cTnI and carotid indices of subclinical atherosclerosis (pINTERACTION $>0.05$ for carotid plaque burden, cIMT and hypoechoic plaques).

Supplementary Table 4 shows discrimination and reclassification models for hs-cTnI according to sex. For women, hs-cTnI improved overall model fit (p for likelihood-ratio test $<0.001)$, NRI $(0.255,95 \%$ CI 0.123 to 0.375$)$ and IDI $(0.009,95 \%$ CI 0.001 to 0.021$)$ in predicting carotid plaques, whereas hs-cTnI improved overall model fit (p for likelihood-ratio test 0.009$)$ and NRI $(0.203,95 \%$ CI 0.001 to 0.336$)$ in predicting cIMT in men.

\section{Discussion}

The principal finding of the current study is that concentrations of cTnI measured with a very high sensitivity assay are associated with carotid atherosclerosis as assessed by total carotid plaque score. Concentrations of hs-cTnI were measurable in practically all study

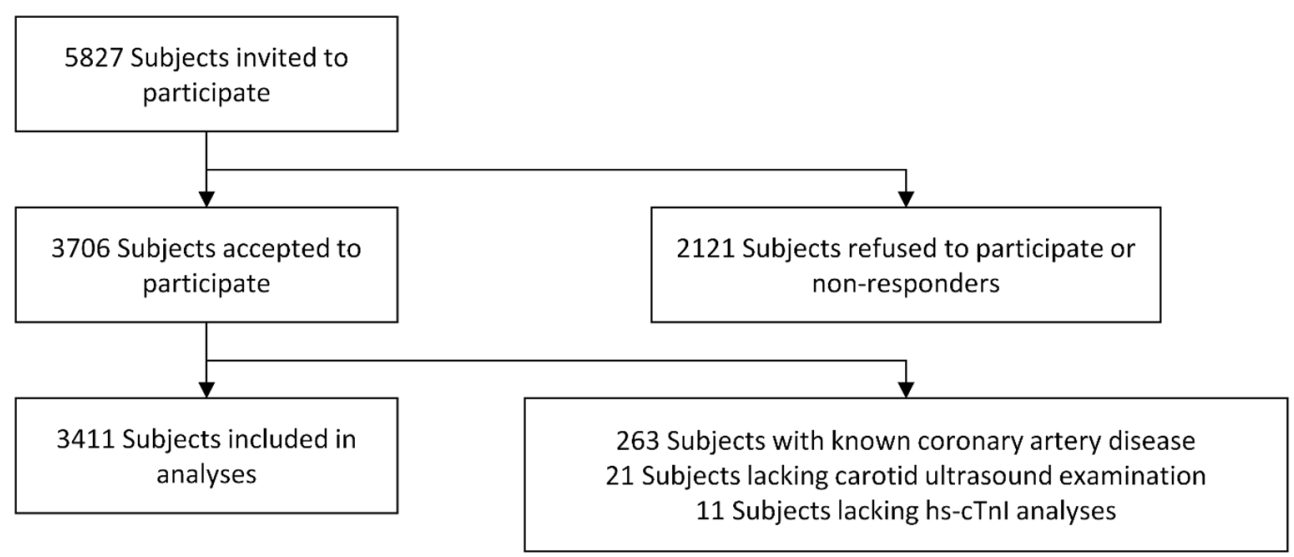

Fig. 1. Flow chart of participation in the study. hs-cTnI, cardiac troponin I. 




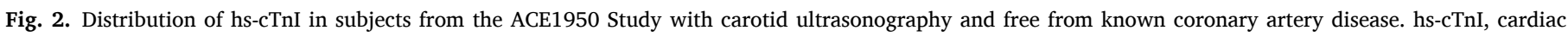
troponin I.

participants and added incremental information to established cardiovascular risk factors in predicting carotid plaque burden.

The use of cardiac troponins as indicators of myocardial injury was implemented in the definition of acute coronary syndromes in 2000. Since then, increasingly sensitive assays have enabled quantification of low-level concentrations of cardiac troponin in large proportions of the general population [25]. cTnI measured by a single-molecule counting method is currently the most sensitive cardiac troponin investigation, with a limit of detection lower than for any other cardiac troponin assay [16]. This is apparent in the current study, as well as in previous reports utilizing the same assay, where measurable concentrations of hs-cTnI were evident in practically all study participants.

Atherothrombosis is the main cause of acute coronary syndromes, and there is a positive association between extent of myocardial injury and cardiac troponin release [26]. In patients with suspected CAD, higher concentrations of hs-cTnT are associated with increased coronary plaque burden and presence of significant coronary stenosis [27]. Higher concentrations of hs-cTnI are in the same way associated with presence and severity of coronary atherosclerosis [28], as well as shortterm mortality and non-fatal coronary events [29]. Analogously to coronary atherosclerosis, quantification of carotid atherosclerosis may provide valuable information on cardiovascular risk. In this regard, carotid plaques may be more sensitive in predicting risk compared to cIMT [30] and both total plaque area and carotid plaque score are predictive of incident coronary heart disease [31]. hs-cTnI predicts overt cerebrovascular disease such as incident ischemic stroke [4] and patients with ischemic stroke commonly exhibit cardiac troponin elevation [5]. cTnI has previously been associated with carotid atherosclerotic plaques in a population based sample [7], with similar associations of hs-cTnT in a smaller cohort of patients with systemic lupus erythematosus [9]. However, both studies were performed with less sensitive cardiac troponin assays, less extensive carotid vascular phenotyping and were underpowered compared to the current investigation. Using the most sensitive cardiac troponin assay currently available, we reaffirm the associations between carotid atherosclerotic burden and subclinical myocardial injury. We additionally elaborate on previous findings by demonstrating significant improvements in reclassification and discrimination models for hs-cTnI in predicting carotid atherosclerosis. Three pathobiological pathways may be responsible for the principal findings of the current investigation:

Firstly, atherosclerosis is a generalized disorder principally affecting all arterial beds and there is significant coexistence of carotid, coronary and peripheral atherosclerosis. $\mathrm{cTnI}$ and $\mathrm{cTnT}$ are expressed specifically in the myocardium and higher concentrations of cardiac troponins are independently associated with coronary atherosclerotic burden [32], possibly due to coronary microembolization and silent ischemic episodes [33]. The association between carotid atherosclerosis and cardiac troponin is most likely a surrogate measure of the association with coexisting coronary atherosclerosis.

Secondly, common risk factors influence atherosclerotic progression in both carotid and coronary arteries [34]. In addition to exerting detrimental effects on the arterial vasculature, diabetes mellitus, hypertension and blood lipids influence the myocardium by pathways other than those mediated by atherosclerotic processes [35]. If left untreated, at-risk subjects may progress into hypertensive and/or diabetic cardiomyopathy, with resulting myocardial fibrosis, left ventricular hypertrophy and ultimately overt heart failure. Cardiac troponins largely predict the presence of myocardial fibrosis [36], and common pathobiological pathways between carotid atherosclerosis and cardiac fibrosis could possibly link carotid plaque burden to concentrations of cardiac troponin.

Thirdly, coronary microvascular dysfunction may have contributed to low-grade release of cardiac troponin observed in the current investigation. Analogously to progressive cardiac fibrosis due to established cardiovascular risk factors such as diabetes mellitus and hypertension, common risk factors precipitate the development of vascular dysfunction even in the absence of overt coronary atherosclerosis. Inferior microvascular function is associated with elevated concentrations of cardiac troponin [37], as well as cardiac troponin increases within the reference range [38]. Subclinical coronary microvascular dysfunction may additionally account for the apparent associations between carotid plaque burden and low-grade increases in hs-cTnI.

With regard to cIMT, the associations with cardiac troponins are more conflicting. In patients with end-stage renal failure, cTnT is associated with carotid atherosclerosis as assessed by cIMT [39]. Similar associations were found for hs-cTnI in the Gutenberg Health Study [40], but not in a large cohort from The Framingham Offspring Study [41]. In the current investigation, we found significant associations between hscTnI and cIMT, but hs-cTnI did not improve reclassification and discrimination models in predicting increased cIMT. cIMT provides limited information on carotid plaque burden [42] and probably serves as an unconvincing proxy of coronary artery atherosclerosis and 
Table 1

Baseline characteristics according to quartiles of hs-cTnI.

\begin{tabular}{|c|c|c|c|c|c|c|c|c|c|c|}
\hline \multirow[t]{2}{*}{ Variable } & \multicolumn{2}{|c|}{ Total cohort } & \multicolumn{2}{|c|}{$\begin{array}{l}\text { Quartile } 1 \text { (hs-cTnI } \leq \\
0.70 \mathrm{ng} / \mathrm{L})\end{array}$} & \multicolumn{2}{|c|}{$\begin{array}{l}\text { Quartile } 2 \text { (hs- } \\
\text { cTnI0.71-1.02 ng/L) }\end{array}$} & \multicolumn{2}{|c|}{$\begin{array}{l}\text { Quartile } 3 \text { (hs- } \\
\text { cTnI1.03-1.61 ng/L) }\end{array}$} & \multicolumn{2}{|c|}{$\begin{array}{l}\text { Quartile } 4 \text { (hs-cTnI } \geq \\
1.62 \mathrm{ng} / \mathrm{L})\end{array}$} \\
\hline & $\mathrm{n}$ & 3411 & $\mathrm{n}$ & 885 & $\mathrm{n}$ & 829 & $\mathrm{n}$ & 855 & $\mathrm{n}$ & 842 \\
\hline Male sex, n (\%) & 3411 & $1666(48.8 \%)$ & 885 & $212(24.0 \%)$ & 829 & $343(41.4 \%)^{* * *}$ & 855 & $484(56.6 \%)^{* * *}$ & 842 & $627(74.5 \%)^{* * *}$ \\
\hline Age, years & 3411 & $\begin{array}{l}63.9(63.4 \text { to } \\
64.4)\end{array}$ & 885 & $\begin{array}{l}63.9(63.5 \text { to } \\
64.4)\end{array}$ & 829 & $\begin{array}{l}63.9(63.4 \text { to } \\
64.5)\end{array}$ & 855 & $\begin{array}{l}63.8(63.4 \text { to } \\
64.4)\end{array}$ & 842 & $\begin{array}{l}63.9(63.4 \text { to } \\
64.5)\end{array}$ \\
\hline Higher education, $\mathrm{n}(\%)$ & 3402 & $1602(47.1 \%)$ & 883 & $380(43.0 \%)$ & 827 & $375(45.3 \%)$ & 853 & $412(48.3 \%)^{*}$ & 839 & $435(51.8 \%)^{* * *}$ \\
\hline Current smoker, n (\%) & 3387 & $483(14.3 \%)$ & 880 & $153(17.4 \%)$ & 825 & $124(15.0 \%)$ & 848 & $127(15.0 \%)$ & 834 & $79(9.5 \%)^{* * *}$ \\
\hline $\begin{array}{l}\text { Alcohol consumption, units } / 2 \\
\text { weeks }\end{array}$ & 3411 & $6(2$ to 11$)$ & 885 & $5(1$ to 10$)$ & 829 & $6(2 \text { to } 11)^{* *}$ & 855 & $6(2 \text { to } 12)^{* * *}$ & 842 & $6(2 \text { to } 12)^{* * *}$ \\
\hline $\begin{array}{l}\text { Study site Akershus University } \\
\text { Hospital, n (\%) }\end{array}$ & 3411 & $2269(66.5 \%)$ & 885 & $578(65.3 \%)$ & 829 & $562(67.8 \%)$ & 855 & $575(67.3 \%)$ & 842 & $554(65.8 \%)$ \\
\hline Body mass index, $\mathrm{kg} / \mathrm{m}^{2}$ & 3411 & $\begin{array}{l}26.6(24.1 \text { to } \\
29.4)\end{array}$ & 885 & $\begin{array}{l}25.7 \text { ( } 23.3 \text { to } \\
28.1)\end{array}$ & 829 & $\begin{array}{l}26.5(23.8 \text { to } \\
29.2)^{* * *}\end{array}$ & 855 & $\begin{array}{l}27.1(24.3 \text { to } \\
29.9)^{* * *}\end{array}$ & 842 & $\begin{array}{l}27.4(24.9 \text { to } \\
30.3)^{* * *}\end{array}$ \\
\hline Heart rate, beats/min & 3410 & 62 (56 to 69$)$ & 885 & 65 (59 to 71$)$ & 829 & $62(57 \text { to } 69)^{* * *}$ & 855 & $61(56 \text { to } 68)^{* * *}$ & 841 & $60(54 \text { to } 66)^{* * *}$ \\
\hline Systolic blood pressure, $\mathrm{mmHg}$ & 3409 & $\begin{array}{l}137 \text { (125 to } \\
149)\end{array}$ & 885 & $\begin{array}{l}132(120 \text { to } \\
144)\end{array}$ & 828 & $\begin{array}{l}135 \text { (125 to } 147) \\
* * *\end{array}$ & 855 & $\begin{array}{l}137 \text { (126 to } 150) \\
* * *\end{array}$ & 841 & $\begin{array}{l}142(129 \text { to } 156) \\
* * *\end{array}$ \\
\hline Diastolic blood pressure, $\mathrm{mmHg}$ & 3409 & 77 (70 to 84$)$ & 885 & 74 (68 to 80$)$ & 828 & $76(70 \text { to } 82)^{* * *}$ & 855 & $78(72 \text { to } 84)^{* * *}$ & 841 & $79(73 \text { to } 87)^{* * *}$ \\
\hline $\begin{array}{l}\text { Diabetes mellitus, n (\%) } \\
\text { Medication }\end{array}$ & 3410 & $265(7.8 \%)$ & 885 & $51(5.8 \%)$ & 828 & $62(7.5 \%)$ & 855 & $62(7.3 \%)$ & 842 & $90(10.7 \%)^{* * *}$ \\
\hline Diuretics, n (\%) & 3411 & $98(2.9 \%)$ & 885 & $13(1.5 \%)$ & 829 & $22(2.7 \%)$ & 855 & $27(3.2 \%)^{*}$ & 842 & $36(4.3 \%)^{* * *}$ \\
\hline$\beta$ blockers, $\mathrm{n}(\%)$ & 3411 & $314(9.2 \%)$ & 885 & $53(6.0 \%)$ & 829 & $71(8.6 \%)^{*}$ & 855 & $90(10.5 \%)^{* * *}$ & 842 & $100(11.9 \%)^{* * *}$ \\
\hline Calcium antagonists, $\mathrm{n}(\%)$ & 3411 & $255(7.5 \%)$ & 885 & $40(4.5 \%)$ & 829 & $56(6.8 \%)^{*}$ & 855 & $80(9.4 \%)^{* * *}$ & 842 & $79(9.4 \%)^{* * *}$ \\
\hline ACE-I/ARB, n (\%) & 3411 & $856(25.1 \%)$ & 885 & $149(16.8 \%)$ & 829 & $197(23.8 \%)$ & 855 & $239(28.0 \%)$ & 842 & $271(32.2 \%)^{* * *}$ \\
\hline Statins, n (\%) & 3411 & $729(21.4 \%)$ & 885 & $160(18.1 \%)$ & 829 & $178(21.5 \%)$ & 855 & $186(21.8 \%)$ & 842 & $205(24.3 \%)^{* *}$ \\
\hline hs-cTnI, ng/L & 3411 & $\begin{array}{l}1.02(0.70 \text { to } \\
1.61)\end{array}$ & 885 & $\begin{array}{l}0.55(0.47 \text { to } \\
0.63)\end{array}$ & 829 & $\begin{array}{l}0.85(0.77 \text { to } \\
0.94)^{* * *}\end{array}$ & 855 & $\begin{array}{l}1.27(1.14 \text { to } \\
1.41)^{* * *}\end{array}$ & 842 & $\begin{array}{l}2.41(1.92 \text { to } \\
3.45)^{* * *}\end{array}$ \\
\hline $\mathrm{CRP} \geq 3 \mathrm{mg} / \mathrm{L}, \mathrm{n}(\%)$ & 3398 & $713(21.0)$ & 880 & $176(20.0 \%)$ & 824 & $182(22.1 \%)$ & 853 & $176(20.6 \%)$ & 841 & $179(21.3 \%)$ \\
\hline HbA1c, $\%$ & 3397 & 5.7 (5.5 to 5.9$)$ & 880 & 5.7 (5.5 to 5.9$)$ & 825 & $5.7(5.5$ to 5.9$)$ & 855 & 5.7 (5.5 to 5.9$)$ & 837 & $5.7(5.5$ to 6.0$)$ \\
\hline Total cholesterol, mmol/L & 3409 & 5.5 (4.8 to 6.2$)$ & 885 & 5.7 (4.9 to 6.4$)$ & 829 & $\begin{array}{l}5.5(4.8 \text { to } 6.1) \\
* * *\end{array}$ & 855 & $\begin{array}{l}5.5(4.7 \text { to } 6.1) \\
* * *\end{array}$ & 840 & $\begin{array}{l}5.4(4.7 \text { to } 6.1) \\
* * *\end{array}$ \\
\hline HDL cholesterol, mmol/L & 3408 & 1.5 (1.2 to 1.8$)$ & 884 & 1.5 (1.3 to 1.9 ) & 829 & 1.5 (1.2 to 1.9$)$ & 855 & $\underset{* * *}{1.5}(1.2$ to 1.8$)$ & 840 & $\begin{array}{l}1.4(1.2 \text { to } 1.8) \\
* * *\end{array}$ \\
\hline LDL cholesterol, mmol/L & 3387 & $3.4(2.7$ to 4.0$)$ & 882 & $3.5(2.9$ to 4.1$)$ & 824 & $\begin{array}{l}3.3(2.8 \text { to } 3.9) \\
* * *\end{array}$ & 848 & $\begin{array}{l}3.3(2.7 \text { to } 3.9) \\
* * *\end{array}$ & 833 & $\begin{array}{l}3.3(2.6 \text { to } 3.9) \\
* * *\end{array}$ \\
\hline Triglycerides, mmol/L & 3408 & $\begin{array}{l}1.17(0.85 \text { to } \\
1.65)\end{array}$ & 885 & $\begin{array}{l}1.13(0.83 \text { to } \\
1.60)\end{array}$ & 829 & $\begin{array}{l}1.17(0.85 \text { to } \\
1.64)\end{array}$ & 855 & $\begin{array}{l}1.14(0.83 \text { to } \\
1.63)\end{array}$ & 839 & $\begin{array}{l}1.21(0.89 \text { to } \\
1.72)^{* *}\end{array}$ \\
\hline eGFR, $\mathrm{mL} / \mathrm{min} / 1.73 \mathrm{~m}^{2}$ & 3395 & $\begin{array}{l}85.0 \text { (75.2 to } \\
92.6)\end{array}$ & 881 & $\begin{array}{l}86.2 \text { (76.3 to } \\
93.1)\end{array}$ & 826 & $\begin{array}{l}85.0 \text { (76.0 to } \\
92.4)\end{array}$ & 851 & $\begin{array}{l}83.8(74.0 \text { to } \\
92.3)^{* *}\end{array}$ & 837 & $\begin{array}{l}84.8(74.4 \text { to } \\
92.3)^{*}\end{array}$ \\
\hline Carotid plaque score & 3411 & $2(1$ to 3$)$ & 885 & $2(1$ to 3$)$ & 829 & $2(1 \text { to } 3)^{*}$ & 855 & $2(1 \text { to } 4)^{* * *}$ & 842 & $2(1 \text { to } 4)^{* * *}$ \\
\hline Carotid plaque score $\geq 4, \mathrm{n}(\%)$ & 3411 & $842(24.7 \%)$ & 885 & $155(17.5 \%)$ & 829 & $184(22.2 \%)^{*}$ & 855 & $242(28.3 \%)^{* * *}$ & 842 & $261(31.0 \%)^{* * *}$ \\
\hline cIMT, mm & 3411 & $\begin{array}{l}0.71(0.65 \text { to } \\
0.79)\end{array}$ & 885 & $\begin{array}{l}0.70(0.64 \text { to } \\
0.77)\end{array}$ & 829 & $\begin{array}{l}0.71(0.64 \text { to } \\
0.79)^{*}\end{array}$ & 855 & $\begin{array}{l}0.72(0.65 \text { to } \\
0.80)^{* * *}\end{array}$ & 842 & $\begin{array}{l}0.74(0.67 \text { to } \\
0.82)^{* * *}\end{array}$ \\
\hline cIMT $>0.90 \mathrm{~mm}, \mathrm{n}(\%)$ & 3411 & $250(7.3 \%)$ & 885 & $40(4.5 \%)$ & 829 & $50(6.0 \%)$ & 855 & $61(7.1 \%)^{*}$ & 842 & $99(11.8 \%)^{* * *}$ \\
\hline Hypoechoic plaques, n (\%) & 3411 & $528(15.5 \%)$ & 885 & $131(14.8 \%)$ & 829 & $119(14.4 \%)$ & 855 & $129(15.1 \%)$ & 842 & $149(17.7 \%)$ \\
\hline
\end{tabular}

p compared to quartile $1: *<0.05, * *<0.01, * * *<0.001$. hs-cTnI, cardiac troponin I. ACE-I, angiotensin-converting-enzyme inhibitor. ARB, angiotensin II receptor

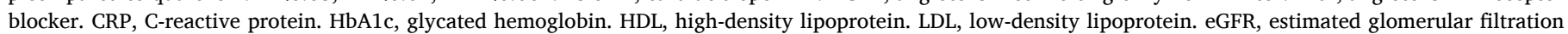
rate. cIMT, carotid intima-media thickness.

subclinical myocardial injury.

We found no associations between hypoechoic plaques and hs-cTnI. Establishing the presence of hypoechoic plaques relies on description of individual plaque morphology [13], and not the very extent of total carotid plaque burden. Put differently, the mere presence of a hypoechoic plaque does not denote significant atherosclerosis and vice versa. Low-grade release of cardiac troponin on the other hand represents subclinical myocardial injury characterized by progressive vascular alterations, fibrosis, and cardiac remodeling [36]. Hypoechoic carotid plaques increase the risk for embolization and stroke compared with echogenic carotid plaques [43], but are not related to such chronic changes in the same way as total carotid plaque burden. The lack of association is expected given the current understanding of these two indices of cardiovascular health in the general population.

In analysis stratified according to sex, we found no effect modification by sex in any of the regression models. Some of the distinct differences by sex do however deserve mentioning. Women exhibited numerically stronger associations between hs-cTnI and carotid plaque burden, and hs-cTnI provided incremental diagnostic information to established cardiovascular risk factor in predicting carotid plaque burden in women. Conversely, the associations of hs-cTnI with cIMT were numerically stronger in men. In the general population, the associations between concentrations of $\mathrm{cTnI}$ and incident myocardial infarction [22] and cardiovascular mortality [23] are stronger in women. Women exhibit lower concentrations of cardiac troponin [24], and for a given absolute value of cardiac troponin, the associated risk would be correspondingly higher. This phenomenon may partly explain the stronger diagnostic impact of hs-cTnI on subclinical atherosclerosis in women.

Several strengths and limitations of the current study merits mentioning. A major strength of the study is the use of the most sensitive cardiac troponin assay developed, in addition to an extensive and highly sensitive bilateral evaluation of the carotid arteries. The study is of greater power than previous investigations on the subject, allowing more precise and sensitive statistical analyses. Concentrations of hs-cTnI were well below the 99th percentile for almost all study participants, and imprecision of the biomarker values in this concentration range may have biased our results. The hs-cTnI values were however predominantly above the $10 \% \mathrm{CV}$ concentration ranges previously reported [16]. We assessed the incremental diagnostic value of hs-cTnI to established cardiovascular risk factors and demographics using multiple statistical indices of discrimination, reclassification and overall model 
Table 2

Associations between hs-cTnI and carotid ultrasound measures.



hs-cTnI, cardiac troponin I. cIMT, carotid intima-media thickness. OR, odds ratio. Model 1, unadjusted. Model 2, adjusted for sex, age and study site. Model 3, adjusted for model 2, BMI, eGFR, total, HDL and LDL cholesterol, CRP, higher education, statin therapy, systolic blood pressure, diabetes mellitus, current smoking, and alcohol consumption.

Table 3

Diagnostic performance of hs-cTnI on carotid ultrasound measures.

\begin{tabular}{|c|c|c|c|}
\hline Model & $\begin{array}{l}\text { Carotid plaque } \\
\text { score } \geq 4\end{array}$ & cIMT $>0.90 \mathrm{~mm}$ & Hypoechoic plaques \\
\hline & \multicolumn{3}{|l|}{ C statistics $(95 \%$ CI) } \\
\hline Basic model & $\begin{array}{l}0.706 \text { ( } 0.685 \text { to } \\
0.726)\end{array}$ & $\begin{array}{l}0.713 \text { (0.679 to } \\
0.747)\end{array}$ & $\begin{array}{l}0.626 \text { ( } 0.600 \text { to } \\
0.652)\end{array}$ \\
\hline $\begin{array}{l}\text { Basic model }+ \text { hs- } \\
\text { cTnI }\end{array}$ & $\begin{array}{l}0.710 \text { ( } 0.690 \text { to } \\
0.730)\end{array}$ & $\begin{array}{l}0.717 \text { ( } 0.683 \text { to } \\
0.751)\end{array}$ & $\begin{array}{l}0.626(0.600 \text { to } \\
0.652)\end{array}$ \\
\hline $\mathrm{p}$ for comparison & $\begin{array}{l}0.049 \\
\text { Net Reclassification In }\end{array}$ & $\begin{array}{l}0.26 \\
\operatorname{dex}(95 \% C I)\end{array}$ & 0.59 \\
\hline \multirow[t]{2}{*}{$\begin{array}{l}\text { Basic model + hs- } \\
\text { cTnI }\end{array}$} & $\begin{array}{l}0.171 \text { ( } 0.084 \text { to } \\
0.247)\end{array}$ & $\begin{array}{l}0.160(-0.103 \text { to } \\
0.289)\end{array}$ & $\begin{array}{l}-0.103(-0.137 \text { to } \\
0.138)\end{array}$ \\
\hline & \multicolumn{3}{|c|}{ Integrated Discrimination Improvement (95\% CI) } \\
\hline $\begin{array}{l}\text { Basic model + hs- } \\
\text { cTnI }\end{array}$ & 0.003 (0 to 0.009$)$ & $\begin{array}{l}0.002(-0 \text { to } \\
0.008)\end{array}$ & $0(-0.001$ to 0.001$)$ \\
\hline
\end{tabular}

hs-cTnI, cardiac troponin I. cIMT, carotid intima-media thickness. Basic model includes sex, age, study site, BMI, eGFR, total, HDL and LDL cholesterol, CRP, higher education, statin therapy, systolic blood pressure, diabetes mellitus, current smoking, and alcohol consumption.

fit. C statistics, NRI and IDI have their advantages and limitations, the former is conservative and often considered insensitive to minor improvements in model prediction [44]. In contrast, NRI and IDI may overinflate the incremental value of novel biomarkers $[45,46]$. This necessitates the use of multiple statistical metrics in contemporary biomarker evaluation. We have used data from a fairly young and large community-based cohort with broad phenotypical characterization, as well as thorough registration of socioeconomic status and medical history. The data is based on self-report and runs the inevitable risk of response bias. We excluded participants with self-reported CAD, and we may accordingly have excluded false-positive subjects without actual coronary substrate. Non-response bias must also be taken in to account, as we do not have information on subjects who actively refused to participate or were inaccessible for study inclusion. Due to the observational nature of the study, we cannot infer causality in any of our analyses. The lack of coronary and myocardial imaging is a major study limitation. Quantification of coronary atherosclerosis by computed tomography angiography and focal and diffuse myocardial scarring by cardiovascular magnetic resonance imaging most certainly would have provided mechanistic insight to the associations between carotid plaque burden and cardiac troponins. Finally, as the study population is almost exclusively northern European Caucasian, our results are not generalizable to subjects of other ethnicities.

In conclusion, concentrations of hs-cTnI measured with a very high sensitivity assay are predictive of carotid atherosclerotic burden and improves risk assessment concerning carotid atherosclerosis. The mechanism for the association will require additional experimental and clinical studies, but our results support the model of common risk factors of subclinical myocardial injury, coronary and carotid atherosclerosis as the cause for the association between hs-cTnI concentrations and carotid plaque burden.

\section{Acknowledgements}

We thank all our study participants for their participation. We also thank our dedicated study staff at the Department of Medical Research, Bærum Hospital, Vestre Viken Hospital Trust and at the Clinical Trial Unit, Division of Medicine, Akershus University Hospital. Parts of the graphical abstract were drawn using Royalty-Free Materials from Colourbox.

\section{Conflicts of interest}

HR has received speaker honoraria from Novartis and Thermo Fisher BRAHMS. HR has received research grants via Akershus University Hospital from EuroDiagnostica AB, HyTest, Biomedica, and Thermo Fisher BRAHMS. TO has received consultancy and speaker honoraria from Abbott Diagnostics, Roche Diagnostics, and Novartis, and research support via Akershus University Hospital from Thermo Fisher BRAHMS, HyTest Ltd., Biomedica, Abbott Diagnostics, Novartis, Singulex, SomaLogic, and Roche Diagnostics. All other authors declare that they have no known competing financial interests or personal relationships that could have appeared to influence the work reported in this paper.

\section{Appendix A. Supplementary data}

Supplementary data to this article can be found online at https://doi. org/10.1016/j.clinbiochem.2021.04.005.

\section{References}

[1] J.T. Saunders, V. Nambi, J.A. de Lemos, L.E. Chambless, S.S. Virani, E. Boerwinkle, R.C. Hoogeveen, X. Liu, B.C. Astor, T.H. Mosley, A.R. Folsom, G. Heiss, J. Coresh, C.M. Ballantyne, Cardiac troponin T measured by a highly sensitive assay predicts coronary heart disease, heart failure, and mortality in the Atherosclerosis Risk in Communities Study, Circulation 123 (2011) 1367-1376.

[2] J.D.W. Evans, S.J.H. Dobbin, S.J. Pettit, E. Di Angelantonio, P. Willeit, Highsensitivity cardiac troponin and new-onset heart failure: a systematic review and meta-analysis of 67,063 patients with 4,165 incident heart failure events, JACC Heart Fail 6 (3) (2018) 187-197.

[3] M. Rienstra, X. Yin, M.G. Larson, J.D. Fontes, J.W. Magnani, D.D. McManus, E. L. McCabe, E.E. Coglianese, M. Amponsah, J.E. Ho, J.L. Januzzi Jr., K.C. Wollert, M.G. Fradley, R.S. Vasan, P.T. Ellinor, T.J. Wang, E.J. Benjamin, Relation between soluble ST2, growth differentiation factor-15, and high-sensitivity troponin I and incident atrial fibrillation, Am. Heart J. 167 (1) (2014) 109-115 e2.

[4] Z. Hijazi, A. Siegbahn, U. Andersson, C.B. Granger, J.H. Alexander, D. Atar, B. J. Gersh, P. Mohan, V.P. Harjola, J. Horowitz, S. Husted, E.M. Hylek, R.D. Lopes, J. J. McMurray, L. Wallentin, A. Investigators, High-sensitivity troponin I for risk assessment in patients with atrial fibrillation: insights from the Apixaban for Reduction in Stroke and other Thromboembolic Events in Atrial Fibrillation (ARISTOTLE) trial, Circulation 129 (6) (2014) 625-634.

[5] J.F. Scheitz, C.H. Nolte, U. Laufs, M. Endres, Application and interpretation of high-sensitivity cardiac troponin assays in patients with acute ischemic stroke, Stroke 46 (4) (2015) 1132-1140.

[6] P.K. Shah, Screening asymptomatic subjects for subclinical atherosclerosis: can we, does it matter, and should we? J. Am. Coll. Cardiol. 56 (2) (2010) 98-105.

[7] K.M. Eggers, L. Lind, H. Ahlstrom, T. Bjerner, C. Ebeling Barbier, A. Larsson, P. Venge, B. Lindahl, Prevalence and pathophysiological mechanisms of elevated 
cardiac troponin I levels in a population-based sample of elderly subjects, Eur. Heart J. 29 (18) (2008) 2252-2258.

[8] C. Andersson, D. Enserro, L. Sullivan, T.J. Wang, J.L. Januzzi Jr., E.J. Benjamin, J. A. Vita, N.M. Hamburg, M.G. Larson, G.F. Mitchell, R.S. Vasan, Relations of circulating GDF-15, soluble ST2, and troponin-I concentrations with vascular function in the community: the framingham heart study, Atherosclerosis 248 (2016) 245-251.

[9] G. Divard, R. Abbas, C. Chenevier-Gobeaux, N. Chanson, B. Escoubet, M. P. Chauveheid, A. Dossier, T. Papo, M. Dehoux, K. Sacre, High-sensitivity cardiac troponin $\mathrm{T}$ is a biomarker for atherosclerosis in systemic lupus erythematous patients: a cross-sectional controlled study, Arthritis Res. Ther. 19 (1) (2017) 132.

[10] G. Korosoglou, S. Lehrke, D. Mueller, W. Hosch, H.U. Kauczor, P.M. Humpert, E. Giannitsis, H.A. Katus, Determinants of troponin release in patients with stable coronary artery disease: insights from CT angiography characteristics of atherosclerotic plaque, Heart 97 (2011) 823-831.

[11] T. Berge, T. Vigen, M.O. Pervez, H. Ihle-Hansen, M.N. Lyngbakken, T. Omland, P. Smith, K. Steine, H. Rosjo, A. Tveit, A.C.E.S. Group, Heart and brain interactionsthe akershus cardiac examination (ACE) 1950 study design, Scand. Cardiovasc. J. 49(6) (2015) 308-315.

[12] H. Ihle-Hansen, T. Vigen, H. Ihle-Hansen, O.M. Ronning, T. Berge, B. Thommessen, M.N. Lyngbakken, E.B. Orstad, S. Enger, S. Nygard, H. Rosjo, A. Tveit, Prevalence of carotid plaque in a 63- to 65-year-old norwegian cohort from the general population: the ACE (Akershus Cardiac Examination) 1950 study, J. Am. Heart Assoc. 7 (10) (2018), e008562.

[13] J.F. Polak, L. Shemanski, D.H. O'Leary, D. Lefkowitz, T.R. Price, P.J. Savage, W. E. Brant, C. Reid, Hypoechoic plaque at US of the carotid artery: an independent risk factor for incident stroke in adults aged 65 years or older, Cardiovasc. Health Study Radiol. 208 (3) (1998) 649-654.

[14] E.B. Mathiesen, K.H. Bonaa, O. Joakimsen, Low levels of high-density lipoprotein cholesterol are associated with echolucent carotid artery plaques: the tromso study, Stroke 32 (9) (2001) 1960-1965.

[15] W.T. Friedewald, R.I. Levy, D.S. Fredrickson, Estimation of the concentration of low-density lipoprotein cholesterol in plasma, without use of the preparative ultracentrifuge, Clin. Chem. 18 (6) (1972) 499-502.

[16] A. Garcia-Osuna, D. Gaze, M. Grau-Agramunt, T. Morris, C. Telha, A. Bartolome, J. J. Bishop, L. Monsalve, R. Livingston, J. Estis, N. Nolan, J. Sandlund, J. Ordonez Llanos, Ultrasensitive quantification of cardiac troponin I by a Single Molecule Counting method: analytical validation and biological features, Clin. Chim. Acta 486 (2018) 224-231.

[17] M.F. Piepoli, A.W. Hoes, S. Agewall, C. Albus, C. Brotons, A.L. Catapano, M.T. Cooney, U. Corra, B. Cosyns, C. Deaton, I. Graham, M.S. Hall, F.D.R. Hobbs, M.L. Lochen, H. Lollgen, P. Marques-Vidal, J. Perk, E. Prescott, J. Redon, D.J. Richter, N. Sattar, Y. Smulders, M. Tiberi, H.B. van der Worp, I. van Dis, W.M.M. Verschuren, S. Binno, E.S.C.S.D. Group, 2016 European Guidelines on cardiovascular disease prevention in clinical practice: The Sixth Joint Task Force of the European Society of Cardiology and Other Societies on Cardiovascular Disease Prevention in Clinical Practice (constituted by representatives of 10 societies and by invited experts) Developed with the special contribution of the European Association for Cardiovascular Prevention \& Rehabilitation (EACPR), Eur. Heart J. 37(29) (2016) 2315-2381.

[18] R.S. Vasan, E.J. Benjamin, M.G. Larson, E.P. Leip, T.J. Wang, P.W.F. Wilson, D. Levy, Plasma natriuretic peptides for community screening for left ventricular hypertrophy and systolic dysfunction: the framing ham heart study, JAMA 288 (10) (2002) 1252-1259.

[19] A.M. Johri, V. Nambi, T.Z. Naqvi, S.B. Feinstein, E.S.H. Kim, M.M. Park, H. Becher, H. Sillesen, Recommendations for the assessment of carotid arterial plaque by ultrasound for the characterization of atherosclerosis and evaluation of cardiovascular risk: from the American society of echocardiography, J. Am. Soc. Echocardiogr. 33 (8) (2020) 917-933.

[20] I. Ford, A.S. Shah, R. Zhang, D.A. McAllister, F.E. Strachan, M. Caslake, D. E. Newby, C.J. Packard, N.L. Mills, High-sensitivity cardiac troponin, statin therapy, and risk of coronary heart disease, J. Am. Coll. Cardiol. 68 (25) (2016) 2719-2728.

[21] S.E. Nissen, S.J. Nicholls, I. Sipahi, P. Libby, J.S. Raichlen, C.M. Ballantyne, J. Davignon, R. Erbel, J.C. Fruchart, J.C. Tardif, P. Schoenhagen, T. Crowe, V. Cain, K. Wolski, M. Goormastic, E.M. Tuzcu, A. Investigators, Effect of very highintensity statin therapy on regression of coronary atherosclerosis: the ASTEROID trial, JAMA 295 (13) (2006) 1556-1565.

[22] M.N. Lyngbakken, H. Rosjo, O.L. Holmen, S. Nygard, H. Dalen, K. Hveem, T. Omland, Gender, high-sensitivity troponin I, and the risk of cardiovascular events (from the Nord-Trondelag Health Study), Am. J. Cardiol. 118 (6) (2016) 816-821.

[23] T. Omland, J.A. de Lemos, O.L. Holmen, H. Dalen, J.S. Benth, S. Nygard, K. Hveem, H. Rosjo, Impact of sex on the prognostic value of high-sensitivity cardiac troponin I in the general population: the HUNT study, Clin. Chem. 61 (2015) 646-656.

[24] F.S. Apple, R. Ler, M.M. Murakami, Determination of 19 cardiac troponin I and T assay 99th percentile values from a common presumably healthy population, Clin. Chem. 58 (2012) 1574-1581.
[25] P. Willeit, P. Welsh, J.D.W. Evans, L. Tschiderer, C. Boachie, J.W. Jukema, I. Ford, S. Trompet, D.J. Stott, P.M. Kearney, S.P. Mooijaart, S. Kiechl, E. Di Angelantonio, N. Sattar, High-sensitivity cardiac troponin concentration and risk of first-ever cardiovascular outcomes in 154,052 participants, J. Am. Coll. Cardiol. 70 (5) (2017) 558-568.

[26] J. Hallen, Troponin for the estimation of infarct size: what have we learned? Cardiology 121 (3) (2012) 204-212.

[27] E.M. Laufer, A.M. Mingels, M.H. Winkens, I.A. Joosen, M.W. Schellings, T. Leiner, J.E. Wildberger, J. Narula, M.P. Van Dieijen-Visser, L. Hofstra, The extent of coronary atherosclerosis is associated with increasing circulating levels of high sensitive cardiac troponin T, Arterioscler. Thromb. Vasc. Biol. 30 (6) (2010) 1269-1275.

[28] F.J. Brunner, F. Kröger, C. Blaum, A. Goßling, T. Lorenz, E. van Erckelens, J. Brätz, D. Westermann, S. Blankenberg, T. Zeller, C. Waldeyer, M. Seiffert, Association of high-sensitivity troponin $\mathrm{T}$ and I with the severity of stable coronary artery disease in patients with chronic kidney disease, Atherosclerosis (2020).

[29] J.L. Januzzi Jr., S. Suchindran, U. Hoffmann, M.R. Patel, M. Ferencik, A. Coles, J. C. Tardif, G.S. Ginsburg, P.S. Douglas, P. Investigators, Single-molecule hsTnI and short-term risk in stable patients with chest pain, J. Am. Coll. Cardiol. 73 (3) (2019) 251-260.

[30] T.Z. Naqvi, M.S. Lee, Carotid intima-media thickness and plaque in cardiovascular risk assessment, JACC Cardiovasc. Imag. 7 (10) (2014) 1025-1038.

[31] C. Mitchell, C.E. Korcarz, A.D. Gepner, J.D. Kaufman, W. Post, R. Tracy, A. J. Gassett, N. Ma, R.L. McClelland, J.H. Stein, Ultrasound carotid plaque features, cardiovascular disease risk factors and events: The multi-ethnic study of atherosclerosis, Atherosclerosis 276 (2018) 195-202.

[32] B. Kvisvik, L. Morkrid, H. Rosjo, M. Cvancarova, A.D. Rowe, C. Eek, B. Bendz, T. Edvardsen, J. Gravning, High-sensitivity troponin T vs I in acute coronary syndrome: prediction of significant coronary lesions and long-term prognosis, Clin. Chem. 63 (2) (2017) 552-562.

[33] R. Erbel, G. Heusch, Coronary microembolization, J. Am. Coll. Cardiol. 36 (1) (2000) 22-24.

[34] L.E. Chambless, A.R. Folsom, V. Davis, R. Sharrett, G. Heiss, P. Sorlie, M. Szklo, G. Howard, G.W. Evans, Risk factors for progression of common carotid atherosclerosis: the atherosclerosis risk in communities study, 1987-1998, Am. J. Epidemiol. 155 (1) (2002) 38-47.

[35] J.R. Sowers, M. Epstein, E.D. Frohlich, Diabetes, hypertension, and cardiovascular disease: an update, Hypertension 37 (4) (2001) 1053-1059.

[36] S.L. Seliger, S.N. Hong, R.H. Christenson, R. Kronmal, L.B. Daniels, J.A.C. Lima, J. A. de Lemos, A. Bertoni, C.R. deFilippi, High-sensitive cardiac troponin T as an early biochemical signature for clinical and subclinical heart failure: MESA (MultiEthnic Study of Atherosclerosis), Circulation 135 (16) (2017) 1494-1505.

[37] V.R. Taqueti, B.M. Everett, V.L. Murthy, M. Gaber, C.R. Foster, J. Hainer, R. Blankstein, S. Dorbala, M.F. Di Carli, Interaction of impaired coronary flow reserve and cardiomyocyte injury on adverse cardiovascular outcomes in patients without overt coronary artery disease, Circulation 131 (6) (2015) 528-535.

[38] S. Takashio, M. Yamamuro, Y. Izumiya, S. Sugiyama, S. Kojima, E. Yamamoto, K. Tsujita, T. Tanaka, S. Tayama, K. Kaikita, S. Hokimoto, H. Ogawa, Coronary microvascular dysfunction and diastolic load correlate with cardiac troponin T release measured by a highly sensitive assay in patients with nonischemic heart failure, J. Am. Coll. Cardiol. 62 (7) (2013) 632-640.

[39] Y. Caliskan, A. Ozkok, T. Akagun, N. Alpay, G. Guz, N. Polat, F. Tufan, T. Ecder, S. Bozfakioglu, Cardiac biomarkers and noninvasive predictors of atherosclerosis in chronic peritoneal dialysis patients, Kidney Blood Press Res. 35 (5) (2012) 340-348.

[40] C. Sinning, T. Keller, T. Zeller, F. Ojeda, M. Schluter, R. Schnabel, E. Lubos, C. Bickel, K.J. Lackner, P. Diemert, T. Munzel, S. Blankenberg, P.S. Wild, S. Gutenberg Health, Association of high-sensitivity assayed troponin I with cardiovascular phenotypes in the general population: the population-based Gutenberg health study, Clin. Res. Cardiol. 103 (3) (2014) 211-222.

[41] D.M. Gopal, M.G. Larson, J.L. Januzzi, S. Cheng, A. Ghorbani, K.C. Wollert, T. Kempf, R.B. D’Agostino, J.F. Polak, V.S. Ramachandran, T.J. Wang, J.E. Ho, Biomarkers of cardiovascular stress and subclinical atherosclerosis in the community, Clin. Chem. 60 (11) (2014) 1402-1408.

[42] N.M. Boulos, J.M. Gardin, S. Malik, J. Postley, N.D. Wong, Carotid plaque characterization, stenosis, and intima-media thickness according to age and gender in a large registry cohort, Am. J. Cardiol. 117 (7) (2016) 1185-1191.

[43] E.B. Mathiesen, K.H. Bonaa, O. Joakimsen, Echolucent plaques are associated with high risk of ischemic cerebrovascular events in carotid stenosis: the Tromsø Study, Circulation 103 (17) (2001) 2171-2175.

[44] M.S. Pepe, K.F. Kerr, G. Longton, Z. Wang, Testing for improvement in prediction model performance, Stat. Med. 32 (9) (2013) 1467-1482.

[45] K.F. Kerr, Z. Wang, H. Janes, R.L. McClelland, B.M. Psaty, M.S. Pepe, Net reclassification indices for evaluating risk prediction instruments: a critical review, Epidemiology 25 (1) (2014) 114-121.

[46] P.M. Burch, W.E. Glaab, D.J. Holder, J.A. Phillips, J.M. Sauer, E.G. Walker, Net reclassification index and integrated discrimination index are not appropriate for testing whether a biomarker improves predictive performance, Toxicol. Sci. 156 (1) (2017) 11-13. 